\title{
Nonhuman Primate Models in Translational Regenerative Medicine
}

\author{
Marcel M. Daadi, Tiziano Barberi, Qiang Shi, and Robert E. Lanford
}

\section{ABSTRACT}

Humans and nonhuman primates (NHPs) are similar in size, behavior, physiology, biochemistry, structure and function of organs, and complexity of the immune system. Research on NHPs generates complementary data that bridge translational research from small animal models to humans. NHP models of human disease offer unique opportunities to develop stem cell-based therapeutic interventions that directly address relevant and challenging translational aspects of cell transplantation therapy. These include the use of autologous induced pluripotent stem cell-derived cellular products, issues related to the immune response in autologous and allogeneic setting, pros and cons of delivery techniques in a clinical setting, as well as the safety and efficacy of candidate cell lines. The NHP model allows the assessment of complex physiological, biochemical, behavioral, and imaging end points, with direct relevance to human conditions. At the same time, the value of using primates in scientific research must be carefully evaluated and timed due to expense and the necessity for specialized equipment and highly trained personnel. Often it is more efficient and useful to perform initial proof-of-concept studies for new therapeutics in rodents and/or other species before the pivotal studies in NHPs that may eventually lead to first-in-human trials. In this report, we present how the Southwest National Primate Research Center, one of seven $\mathrm{NIH}$-funded National Primate Research Centers, may help the global community in translating promising technologies to the clinical arena.

\section{Introduction to Texas Biomed and SNPRC}

The Texas Biomedical Research Institute (Texas Biomed) is a leading nonprofit institution dedicated to advancing human health through basic biomedical research. Founded in 1941, it is composed of two research departments, the Department of

Southwest National Primate Research Center, Texas Biomedical Research Institute, San Antonio, Texas.

*(Correspondence: mdaadi@txbiomed.org)
Genetics and the Department of Virology and Immunology, and the Southwest National Primate Research Center (SNPRC). Texas Biomed has major programs in several areas of regenerative medicine and stem cell research supported by various nonhuman primate (NHP) colonies maintained at SNPRC.

The Department of Genetics focuses on characterizing the genetic components of susceptibility to common complex diseases through studies of human and NHP populations. Cardiovascular disease, diabetes, obesity, fetal growth and development, osteoporosis, epilepsy, and psychiatric disease are among the many well-established research areas for this group. The department houses one of the world's largest computer clusters dedicated to genetic analysis of human and NHP data. The cluster is housed in the AT\&T Genomics Computing Center, which currently contains 8,000 processors.

The Department of Virology and Immunology focuses on a broad array of human infectious diseases, including AIDS, hepatitis, and herpes virus infections. In addition, there is a research 
emphasis on high-containment agents with potential to be bioterror agents due to their high morbidity and mortality in humans, such as Ebola and Marburg viruses, SARS, avian flu, and anthrax. The work on these agents is performed at biosafety level 4 (BSL-4). Texas Biomed established one of the first non-governmentowned BSL-4 laboratories and is a leader in vaccine development for select agents.

The Southwest National Primate Research Center (SNPRC) is one of seven NIHfunded National Primate Research Centers. The SNPRC maintains breeding colonies of three primate species for use in biomedical research, and can obtain additional species as needed to meet research demands. The SNPRC baboon colony has over 1500 animals and includes one of the most extensively characterized NHP pedigrees available for study. The baboon pedigree covers seven generations of animals, which have been assessed for genetic variations influencing risk factors for common complex diseases such as heart disease, obesity, and osteoporosis. Together, the pedigreed colony and the baboon gene map give scientists incredibly powerful research tools for finding the genes that underlie natural susceptibility to, or protection from, a variety of diseases. Because of the ability to control dietary and other environmental factors in studies of the baboons, the Department of Genetics has used the pedigreed baboon colony to assess the interactive effects of diet and genotype on cardiovascular disease. The rhesus macaque colony is an NIH-funded, specific pathogen-free colony, which is used primarily to supply animals to support AIDS research. The marmoset colony is a unique resource for areas of regenerative medicine, aging, obesity and diabetes, and infectious diseases. Marmosets reach reproductive maturity at 15 months and can give birth to twins twice per year. This small New World primate is particularly attractive for stem cell work and transgenics.

SNPRC Pilot Funding Opportunities in Regenerative Medicine: The SNPRC Pilot Research program provides opportunities for investigators to use the resources of the SNPRC in highly focused, short-term studies. Priority is given to studies that will generate preliminary data to support future NIH grant applications. Pilot grants normally are awarded for periods of 1-2 years and provide a maximum of $\$ 100,000$ for use in primate-related research. The program accepts applications once or twice per year depending upon availability of funds (see www.txbiomed.org/primate -research-center/pilot-study-funding for details). Examples of funded research in the area of regenerative medicine include stem cell therapy in the marmoset using intranasal delivery, cognitive dysfunction
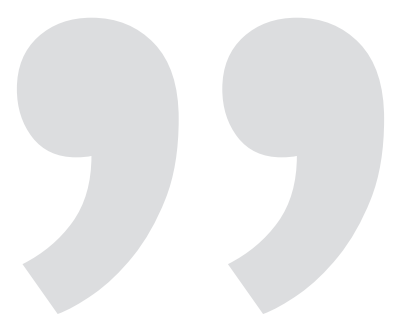

The SNPRC Pilot

Research program provides opportunities for investigators conducting highly focused, short-term studies.

in the marmoset experimental autoimmune encephalomyelitis model, transplantation of cultured baboon spermatogonial stem cells, and integrating motor and cognitive measures in a Parkinson's disease (PD) model.

\section{Animal models for stem} cell-based regenerative medicine

Humans and NHPs are similar in their size, behavior, physiology, biochemistry, structure and function of organs, and complexity of the immune system. Research on NHPs can generate complementary data that bridge translational research from small animal models to humans. NHP models of human disease offer unique opportunities to develop stem cell-based therapeutic interventions that directly address relevant and known translational aspects of cell transplantation therapy. These include the use of autologous induced pluripotent stem cell (iPSC)-derived cellular products, issues related to the immune response in autologous and allogeneic setting, pros and cons of delivery techniques in a clinical setting, as well as the safety and efficacy of candidate cell lines. The NHP model allows the assessment of complex physiological, biochemical, behavioral, and imaging end points, with direct relevance to human conditions.

For example, in PD, the use of the neurotoxin 1-methyl-4-phenyl-1,2,3,6tetrahydropyridine (MPTP) to create an NHP disease model of PD offers important preclinical research opportunities; the model will be essential to demonstrate clinically relevant safety and efficacy data prior to first-in-human trials. As in humans $[1,2]$, MPTP injections in NHPs replicate most features of sporadic PD, including static tremor [3], one of the most difficult symptoms and one that can be observed only in some monkey models of PD. Furthermore, as experienced by humans with PD, MPTP-treated NHPs develop side effects to anti-parkinsonian drugs such as dyskinesias in the case of LevoDOPA pharmacotherapy [4]; cumulate the major constituent, a-synuclein, of Lewy bodies, the pathological hallmark of PD [5]; and express cognitive disturbances [6]. The NHP models for PD have already contributed significantly to our understanding of the autologous versus allogeneic approaches to PSC-based therapies, the appropriate differentiation state of the dopaminergic neurons, and their capacity to reinnervate the putamen [7-13].

The SNPRC has projects focused on a variety of stem cell-based regenerative therapies for neurological disorders. These 
FIG. 1. Primate stem cell technologies in regenerative medicine and drug discovery: Induced pluripotent stem cell (iPSC) lines can be derived from adult somatic cell biopsies taken directly from patients or nonhuman primates, or from archived specimens in tissue banks. These tissue or blastocyst-derived pluripotent stem cells may be genetically engineered with targeted mutations or targeted corrections of defective genes, then differentiated into organ-specific functional cell types. This approach offers powerful cell based assays that may improve the specialization of tissue engineering processes for autologous or allogeneic stem cell based therapies. These assays may also improve the drug development process and provide a platform for understanding diseases at the cellular and molecular level.

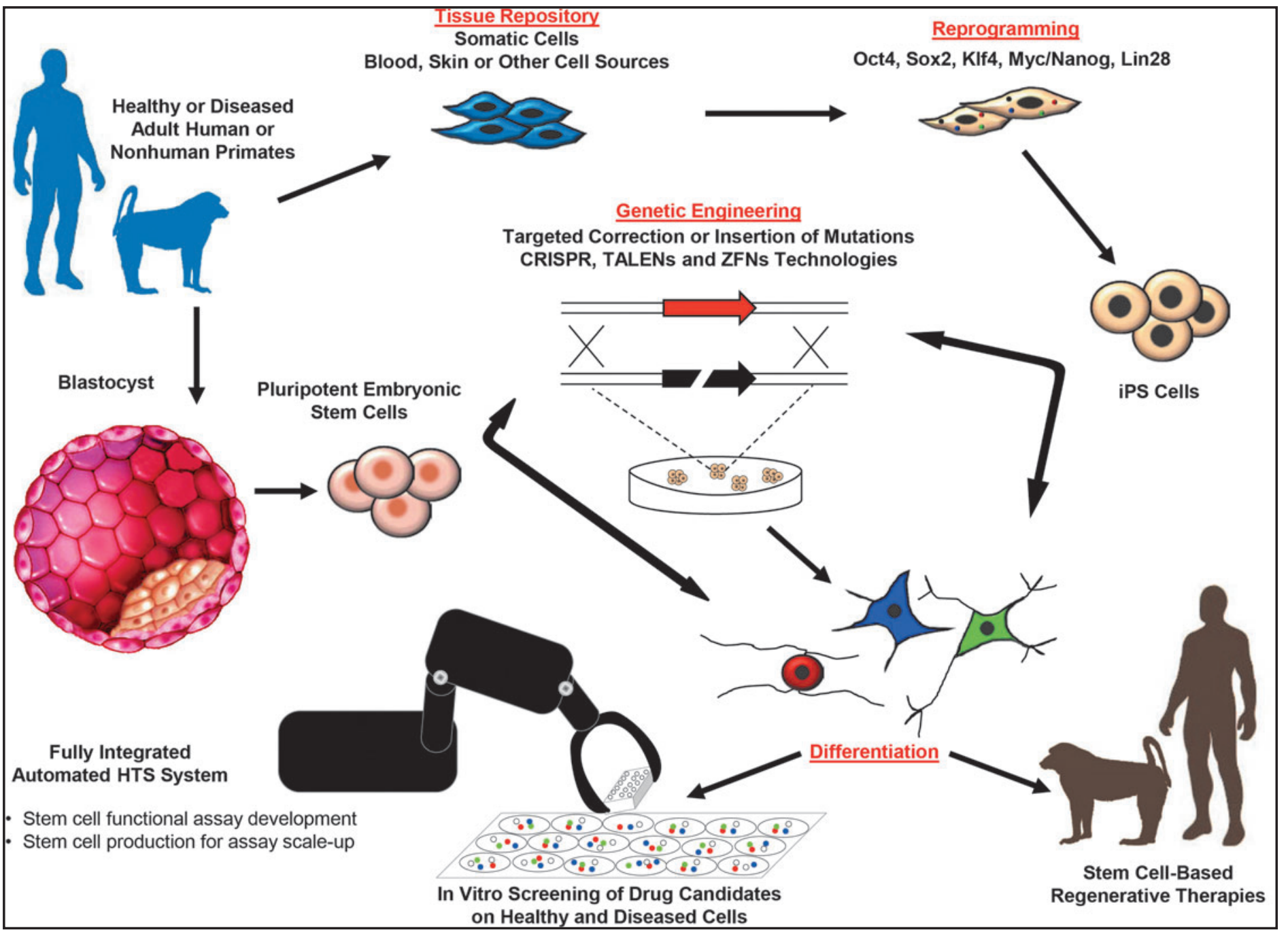

efforts will produce translational data that can demonstrate therapeutic potential and to identify clinical hurdles that must be overcome. In phase I studies, PD patients with neural grafts have experienced improvement in their quality of life for many years $[14,15]$. Thus, the proof of concept has been demonstrated; however, the next challenge is to focus on the development of predictable delivery system and a viable stem cell product that meets strict regulatory requirements to ensure the safety and efficacy of the treatment and eventually successfully move the product toward commercialization and use as a standardized treatment.

Stroke is another area in which NHP models can provide important information on pathophysiology that complements data generated from rodent models of stroke. The importance of NHP models has been highlighted by the Stroke Therapy Academic Industry Roundtable in a set of guidelines that note the need for NHP models of stroke in the preclinical development of neuroprotective and restorative therapies [16]; the guidelines were prompted by the disappointing and unanticipated clinical results of neuroprotective agents that showed efficacy in rodent models. Critical physiological aspects of stroke are unique to NHPs and humans, such as the subcortical white matter injury, including the internal capsule where the descending cortical pathways are located. Similarly, the fine motor skills affected by stroke are controlled by the 
direct cortico-motorneuronal pathway, which is more developed in humans and NHPs than in rodents. NHP models of stroke with exquisite imaging, pathophysiological, and behavioral characterization already have been described [17-23], and recent studies illustrate the utility of such models in preclinical development of therapeutic intervention [24-26]. The ultimate goal of safety and efficacy studies in NHP models is to better predict outcomes, address challenges, and increase the likelihood of success in human clinical trials.

Ongoing studies at the SNPRC are working on the directed differentiation of PSCs into skeletal muscle, retinal progenitors, and lens epithelium. Early muscle progenitors isolated by fluorescence-activated cell sorting (FACS) and able to terminally differentiate into mature muscle cells [27] will be utilized for translational studies in animal models, including NHPs. Although NHP models of muscle disease are not available, an artificial injury model will be tested to evaluate functional competence of PSCderived muscle progenitors to repair damaged muscle fibers. Human PSCs are also differentiated toward lens epithelium [28] or retinal progenitors. The resulting cells purified by FACS are going to be grafted in NHP models of cataract or retinal injury. The high accessibility of the eye and minimal invasive procedure will enable investigators to evaluate, first, integration of cells in the host, and then, using a variety of behavioral tests, the ability of grafted cells to ameliorate or restore vision. Those procedures will expand SNPRC capabilities and will help to establish the NHP model as an elective model for preclinical studies.

The SNPRC also has research ongoing studying the deterioration of the vascular endothelium, a critical condition of vascular disorders. Baboon embryonic stem cells (bESCs) are used for deriving endothelial progenitors to reconstruct experimentally damaged arterial vessels [29]. The investigators are developing technologies to engineer vascular tissue by driving vascular progenitors into functional conduits. Pluripotent baboon stem cells are uniquely suited for engineering vascular tissue because they can generate unlimited numbers of specialized cells in the vessels. The goal of this work is to establish the feasibility of engineering fully viable vascular tissue from bESCs $(2-3 \mathrm{~cm}$ in length and 3$4 \mathrm{~mm}$ in diameter) and to transplant this engineered tissue into a baboon in order to examine its patency and evaluate shortterm therapeutic potential. In addition, SNPRC has developed a baboon model with balloon catheter-induced vascular injury to test the safety and engraftability of endothelial progenitors derived from bESCs and establish the feasibility of this potential cell therapeutic approach.

Given the close phylogenetic, immunologic, anatomic, and physiologic similarities to humans, NHP models of human disease are necessary for understanding basic biological mechanisms and for many translational regenerative medicine applications. At the same time, the value of using primates in scientific research must be carefully evaluated and timed due to expense and the necessity for specialized equipment and highly trained personnel. Often it is more efficient and useful to perform initial proof-of-concept studies for new therapeutics in rodents and/or other species before the pivotal studies in NHPs that may eventually lead to first-in-human trials.

\section{ACKNOWLEDGMENTS}

We thank Dr. Sarah Williams-Blangero for comments on this article.

\section{Author Disclosure} Statement

All authors declare that no competing financial interests exist.

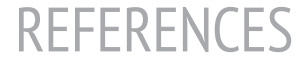

1. Davis GC, AC Williams, SP Markey, MH Ebert, ED Caine, et al. (1979).
Chronic Parkinsonism secondary to intravenous injection of meperidine analogues. Psychiatry Res 1:249-254.

2. Langston JW, P Ballard, JW Tetrud and I Irwin. (1983). Chronic Parkinsonism in humans due to a product of meperidineanalog synthesis. Science 219:979-980.

3. Bergman H, A Raz, A Feingold, A Nini, I Nelken, et al. (1998). Physiology of MPTP tremor. Mov Disord 13 Suppl 3:29-34.

4. Bedard PJ, T Di Paolo, P Falardeau and R Boucher. (1986). Chronic treatment with L-DOPA, but not bromocriptine induces dyskinesia in MPTP-parkinsonian monkeys. Correlation with [3H]spiperone binding. Brain Res 379:294-299.

5. Purisai MG, AL McCormack, WJ Langston, LC Johnston and DA Di Monte. (2005). Alpha-synuclein expression in the substantia nigra of MPTP-lesioned nonhuman primates. Neurobiol Dis 20:898906.

6. Schneider JS and CJ Kovelowski 2nd. (1990). Chronic exposure to low doses of MPTP. I. Cognitive deficits in motor asymptomatic monkeys. Brain Res 519:122-128.

7. Emborg ME, Y Liu, J Xi, X Zhang, Y Yin, et al. (2013). Induced pluripotent stem cell-derived neural cells survive and mature in the nonhuman primate brain. Cell Rep 3:646-650.

8. Daadi MM, BA Grueter, RC Malenka, DE Redmond Jr. and GK Steinberg. (2012). Dopaminergic neurons from midbrain-specified human embryonic stem cell-derived neural stem cells engrafted in a monkey model of Parkinson's disease. PLoS ONE 7:e41120. 9. Takagi Y, J Takahashi, H Saiki, A Morizane, T Hayashi, et al. (2005). Dopaminergic neurons generated from monkey embryonic stem cells function in a Parkinson primate model. J Clin Invest 115:102-109.

10. Kriks S, JW Shim, J Piao, YM Ganat, DR Wakeman, et al. (2011). Dopamine neurons derived from human ES cells efficiently engraft in animal models of Parkinson's disease. Nature 480:547-551. 11. Morizane A, D Doi, T Kikuchi, K Okita, A Hotta, et al. (2013). Direct comparison of autologous and allogeneic transplantation of iPSC-derived neural 
cells in the brain of a nonhuman primate. Stem Cell Rep 1:283-292.

12. Doi D, A Morizane, T Kikuchi, H Onoe, T Hayashi, et al. (2012). Prolonged maturation culture favors a reduction in the tumorigenicity and the dopaminergic function of human ESC-derived neural cells in a primate model of Parkinson's disease. Stem Cells 30:935-945.

13. Emborg ME, Z Zhang, V Joers, $K$ Brunner, V Bondarenko, et al. (2013). Intracerebral transplantation of differentiated human embryonic stem cells to hemiparkinsonian monkeys. Cell Transplant 22:831-838.

14. Kefalopoulou Z, M Politis, P Piccini, N Mencacci, K Bhatia, et al. (2013). Long -term clinical outcome of fetal cell transplantation for Parkinson disease: two case reports. JAMA Neurol. 71:83-87.

15. Mendez I, A Vinuela, A Astradsson, K Mukhida, P Hallett, et al. (2008). Dopamine neurons implanted into people with Parkinson's disease survive without pathology for 14 years. Nat Med 14:507509.

16. STAIR. (1999). Recommendations for standards regarding preclinical neuroprotective and restorative drug development. Stroke 30:2752-2758.

17. Marshall JW and RM Ridley. (2003). Assessment of cognitive and motor deficits in a marmoset model of stroke. Ilar J 44:153-160.

18. Freret T, V Bouet, J Toutain, $\mathrm{R}$ Saulnier, P Pro-Sistiaga, et al. (2008). Intraluminal thread model of focal stroke in the non-human primate. J Cereb Blood Flow Metab 28:786-796.

19. Fukuda S and GJ del Zoppo. (2003). Models of focal cerebral ischemia in the nonhuman primate. Ilar J 44:96-104.

20. Nudo RJ, D Larson, EJ Plautz, KM Friel, S Barbay, et al. (2003). A squirrel monkey model of poststroke motor recovery. Ilar J 44:161-174.

21. Huang J, J Mocco, TF Choudhri, A Poisik, SJ Popilskis, et al. (2000). A modified transorbital baboon model of reperfused stroke. Stroke 31:3054-3063.

22. Kuge Y, C Yokota, M Tagaya, Y Hasegawa, A Nishimura, et al. (2001). Serial changes in cerebral blood flow and flow-metabolism uncoupling in primates with acute thromboembolic stroke. J Cereb Blood Flow Metab 21:202-210.

23. Virley D, SJ Hadingham, JC Roberts, B Farnfield, H Elliott, et al. (2004). A new primate model of focal stroke: endothelin1-induced middle cerebral artery occlusion and reperfusion in the common marmoset. J Cereb Blood Flow Metab 24:24-41.

24. Bahjat FR, RL Williams-Karnesky, SG Kohama, GA West, KP Doyle, et al.
(2011). Proof of concept: pharmacological preconditioning with a Toll-like receptor agonist protects against cerebrovascular injury in a primate model of stroke. J Cereb Blood Flow Metab 31:1229-1242.

25. Cook DJ, L Teves and M Tymianski. (2012). Treatment of stroke with a PSD-95 inhibitor in the gyrencephalic primate brain. Nature 483:213-217.

26. Roitberg BZ, E Mangubat, EY Chen, K Sugaya, KR Thulborn, et al. (2006). Survival and early differentiation of human neural stem cells transplanted in a nonhuman primate model of stroke. J Neurosurg 105:96-102.

27. Borchin B, J Chen and T Barberi. (2013). Derivation and FACS-mediated purification of $\mathrm{PAX} 3+/ \mathrm{PAX} 7+$ skeletal muscle precursors from human pluripotent stem cells. Stem Cell Rep 1:620-631.

28. Mengarelli I and T Barberi. (2013). Derivation of multiple cranial tissues and isolation of lens epithelium-like cells from human embryonic stem cells. Stem Cells Transl Med 2:94-106.

29. Shi Q, G Schatten, V Hodara, C Simerly and JL VandeBerg. (2013). Endothelial reconstitution by $\mathrm{CD} 34+$ progenitors derived from baboon embryonic stem cells. J Cell Mol Med 17:242-251. 